\title{
Connaissances et pratiques ethnobotaniques en médecines traditionnelles vétérinaire et humaine au Bénin : similarité ou dissemblance?
}

\author{
*OUACHINOU Jéronime M-A. S., ADOMOU Aristide C., DASSOU G. Hospice, YEDOMONHAN \\ Hounnankpon, TOSSOU G. Monique et AKOEGNINOU Akpovi \\ Laboratoire de Botanique et Écologie Végétale (LaBEV), Faculté des Sciences et Techniques (FAST), Université \\ d'Abomey-Calavi, Bénin, 01 BP 4521 Cotonou \\ *Auteur correspondant, E-mail : ouajeronime@gmail.com Tel : (+229) 96926363
}

Original submitted in on 23rd June 2016. Published online at www.m.elewa.org on 31st May 2017 https://dx.doi.org/10.4314/jab.v113i1.6

\section{RÉSUMÉ}

Objectif : Rechercher la similarité entre les usages liés aux plantes sollicitées en ethnomédecines vétérinaire et humaine afin de contribuer à la valorisation de ces connaissances endogènes dans la zone cotonnière du centre-Bénin.

Méthodes et Résultats : Des enquêtes ont été réalisées individuellement auprès de 105 éleveurs, agroéleveurs et guérisseurs traditionnels. Les paramètres de diversité floristique, ceux relatifs aux pratiques ethnobotaniques et les facteurs socioculturels déterminant la connaissance ethnobotanique sont utilisés pour les diverses comparaisons basées sur des tests de Khi Deux de Pearson et de deux proportions. La fréquence (S) d'espèces ou d'usages communs a été calculée pour évaluer le taux de similarité entre les deux types de médecine. Quatre-vingt douze (92) espèces de plantes impliquées dans la composition de 122 recettes ont été recensées pour les deux types de médecines. Globalement, la similarité est très grande entre les deux types de médecine en termes de plantes utilisées $(86,69 \%)$ et grande en termes d'usages rapportés $(65 \%)$.

Conclusion et applications des résultats : Certaines connaissances ethnovétérinaires proviendraient de la médecine humaine et vice-versa. Les plantes efficaces utilisées en ethnomédecine vétérinaire pourraient être indiquées dans le traitement des mêmes maladies chez l'homme en cas d'une similarité d'agents pathogènes. Les études phytochimiques, pharmacologiques et cliniques devraient être envisagées pour la valorisation effective de ces plantes.

Mots clés. Ethnomédecines vétérinaire et humaine, origine, similarité, Centre-Bénin.

\section{ABSTRACT}

Objective: To look for similarities between practices related to plants used in veterinary and human ethnomedicines in order to contribute to the promotion of such indigenous knowledge in the cotton zone of Benin center.

Methodology and Results: Surveys were conducted through individual interviewees with 105 breeders and traditional healers in Central Benin. The parameters of floristic diversity, those relating to ethnobotanical practices and sociocultural factors determining ethnobotanical knowledge, were used for various comparisons based on Pearson's Chi squared and two proportions tests. Frequency (S) of common species and reported 
uses was calculated to evaluate the similarity degree between two types of medicines. Ninety two (92) species of plants involved in the composition of 122 recipes were identified for both types of medicine. Overall, the similarity is quite high between the two types of medicines in terms of plants used ( $86.69 \%)$ and high in terms of reported uses $(65 \%)$.

Conclusion and Applications of findings: Part of ethnoveterinary knowledge stems from traditional human medicine, and vice versa. Effective plants used in ethnoveterinary medicine could be indicated for the treatment of the same diseases in human in case of similarity of pathogens. Phytochemical, pharmacological and clinical studies should be considered for the effective valorisation of these plants.

Keywords. Veterinary and human ethnomedicines, origin, similarity, Center of Benin.

\section{INTRODUCTION}

Depuis des siècles, les éleveurs et les guérisseurs africains ont accumulé des connaissances ethnobotaniques pour améliorer les soins animaux et humains. Ces connaissances sont transmises oralement au sein de la société et leur contenu demeure un patrimoine soit d'une famille, soit d'un groupe social particulier du village ou de la contrée (Tamboura et al., 1998). Ainsi, chaque peuple possède son recueil de recettes de médecine traditionnelle dans leur état brut et naturel. Aujourd'hui, la transmission du savoir-faire endogène se détériore peu à peu avec l'évolution de la médecine moderne alors que l'ethnomédecine joue encore un rôle important, en particulier dans les milieux ruraux en complétant les services vétérinaires et de santé humaine. Plusieurs études ethnobotaniques se sont évertuées depuis plusieurs années à identifier les plantes médicinales utilisées pour traiter divers pathologies et symptômes chez l'Homme et à tester leur efficacité par le biais de recherches phytochimiques et pharmacologiques. Ces plantes traitent-elles les mêmes maladies chez les animaux? Des approches théoriques (Scarpa, 2000 ; Lans et al., 2000 ; McCorkle et Martin, 1998 ; Schillhorn van Veen, 1998) et analytiques (Martínez

\section{MATERIEL ET METHODES}

Milieu d'étude : Les études ont été conduites dans la zone cotonnière du Centre-Bénin (figure 1). La moyenne annuelle de la température est $28,26^{\circ} \mathrm{C}$ pour la période de 2000 à 2009. Le climat est de type tropical humide de transition avec une pluviométrie moyenne annuelle de $1107,99 \mathrm{~mm}$ d'eau (Akoègninou, 2004). La période sèche couvre 5 mois (novembre à mars) et la période humide s'étend sur le reste de l'année. Sur le plan de la végétation, le Centre-Bénin appartient à la zone soudano- et Luján, 2011) ont montré que les diverses formes d'usage ou les pratiques médicales d'une part sont similaires dans les deux médecines et d'autre part que les parties de plantes utilisées, les voies d'administration des médicaments en ethnomédecine vétérinaire sont basées sur l'ethnomédecine humaine. En Afrique, aucune étude du genre n'a été menée pour comprendre les relations qui existent entre les deux types de médecine. Or, la compréhension de cette relation permettrait de valoriser la complémentarité entre les deux types de médecine. C'est dans ce cadre que s'inscrit la présente étude intitulée: "Connaissances et pratiques ethnobotaniques en médecines traditionnelles vétérinaire et humaine au Bénin : similarité ou dissemblance? ». Cette étude suppose que les connaissances et pratiques endogènes des populations béninoises en matière de la médecine vétérinaire proviendraient de celles utilisées en ethnomédecine humaine. Son objectif est de rechercher la similarité entre les deux types de médecines en termes de connaissance et pratiques sur les plantes utilisées afin de mettre en valeur la complémentarité qui existe entre les médecines traditionnelles vétérinaire et humaine.

guinéenne dont la végétation est une mosaïque de savanes, de forêts claires parsemées de galeries forestières et des champs et plantations (Akoègninou et al., 2006). La population est de 268.356 habitants soit 67 habitants par km² (INSAE, 2013). Les groupes ethniques présents sont : Fon et Mahi (58\%); Yoruba, Ifè, Itcha et Idaatcha (32\%), Yom-lokpa (2,3\%), Peulh $(2,2 \%)$, Ottamari $(2,5 \%)$, Adja, Dendi et Bariba (3\%) (INSAE, 2002). L'agriculture est la première activité de la 
population et les activités secondaires sont : le commerce et la chasse. La zone d'étude montre une concentration plus élevée en poulets avec une densité de 1422 têtes $/ \mathrm{km}^{2}$. Viennent ensuite, les cheptels de caprins (68 têtes $/ \mathrm{km}^{2}$ ), d'ovins (24 têtes $/ \mathrm{km}^{2}$ ), de porcins (24 têtes $/ \mathrm{km}^{2}$ ), de bovins (5 têtes $\left./ \mathrm{km}^{2}\right)$, (DE, 2010).

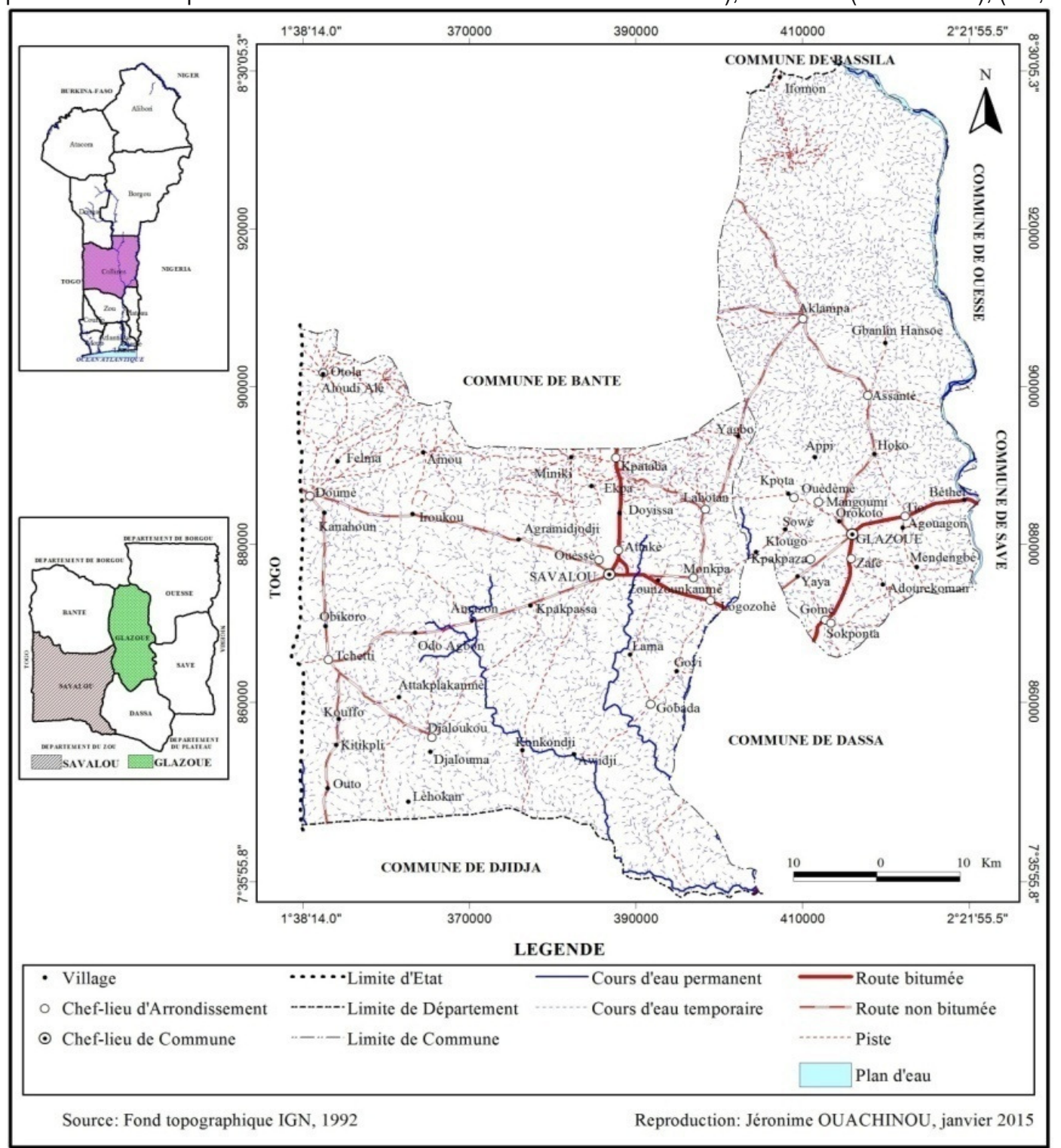

Figure 1 : Carte du Bénin et celle de la zone d'étude montrant les localités prospectées

Enquêtes ethnobotaniques : Trois types d'individus ont été enquêtés dans les communes de Savalou et de Glazoué qui concentrent la taille la plus élevée du cheptel au Centre-Bénin. II s'agit : des éleveurs et agroéleveurs, $\mathrm{du}$ fait de leur connaissance en ethnomédecine vétérinaire ; et des guérisseurs traditionnels à cause de leur aptitude dans le traitement traditionnel des maladies. Au total, 8 villages et 105 individus ont été choisis avec l'aide des Responsables du Développement Rural (RDR) et des Chefs d'Arrondissement suivant les critères définis par van den Eynden et al. (1993) à savoir : la célébrité dans la pratique de la médecine traditionnelle, la réputation ou la popularité du village en médecine traditionnelle, l'usage exclusif ou majoritaire des produits végétaux ou naturels dans le processus de guérison. A ces critères, y est ajouté un autre défini par Dassou et al. (2015) à savoir: la concentration élevée d'animaux pendant la période de l'enquête qui s'étend de juin à août 2014. Les entretiens ont été effectués individuellement par le biais de questionnaires. Les données collectées 
sont relatives au profil des enquêtés (ethnie, sexe, âge, niveau d'instruction), aux maladies traitées, aux noms vernaculaires des plantes utilisées, aux organes prélevés, aux méthodes de préparation et aux modes d'administration des médicaments. Les classes d'âges ont été définies par modification de celles proposées par Gnanglè et al. (2012). Les échantillons végétaux ont été collectés et herborisés. L'identification taxonomique a été réalisée à l'Herbier National du Bénin par comparaison avec les herbiers de référence et à l'aide de la Flore Analytique du Bénin (Akoègninou et al., 2006) dont la nomenclature a été suivie.

Afin de mieux cerner la similarité entre les deux types de médecine, des pathologies communes (gastro- intestinales et dermatologiques) à l'homme et aux animaux ont été considérées, par exemple : la diarrhée, la constipation, la gale, l'envenimation, les douleurs abdominales. Leur choix est basé sur des travaux antérieurs (Dassou et al., 2014 ;Guézodjè, 2013 ; Hounzangbé-Adoté, 2001) ayant montré leur prévalence élevée dans les élevages au Bénin. La répartition des enquêtés suivant les caractéristiques socio-culturelles est présentée dans le tableau 1. Les Peulh étaient les plus enquêtés avec un taux de $35 \%$ de l'échantillon. Les enquêtés étaient en majorité des hommes $(89,52 \%)$ et à $71,43 \%$ non instruits. Leur âge variait entre 25 et 95 ans avec une moyenne de 46 ans. La majorité (59\%) avait entre 40 et 60 ans.

Tableau 1 : Répartition des enquêtés suivant les caractéristiques socioculturelles

\begin{tabular}{lcc}
\hline Variables & Nombres d'enquêtés & Fréquences (\%) \\
\hline Ethnie & 37 & 35 \\
Peulh & 28 & 27 \\
Mahi & 30 & 29 \\
Ifè & 10 & 09 \\
Idaatcha & & \\
Sexe & 11 & 89,52 \\
Masculin & 94 & 10,48 \\
Féminin & & \\
Classes d'âges & 33 & 31,43 \\
Jeunes (âges <40ans) & 59 & 56,19 \\
Adultes (40 Sâges <60ans) & 13 & 12,38 \\
Âgés (60ans Sâges) & & \\
Niveau d'instruction & 75 & 71,43 \\
Non instruits & 20 & 19,05 \\
Niveau primaire & 9 & 8,57 \\
Niveau secondaire & 1 & 0,95 \\
Niveau universitaire & & \\
Origine de la connaissance & 52 & 42,28 \\
Amis éleveurs & 64 & 52,03 \\
Famille & 5 & 4,07 \\
Formation & 2 & 1,62 \\
Institution d'élevage & 105 & 100 \\
TOTAL & & \\
& &
\end{tabular}

Traitement des données : Des calculs de fréquence ont été effectués pour diverses variables (plantes, maladies, organes utilisés, modes de préparation et voies d'administration). Pour analyser les liens entre les différents paramètres floristiques (richesse spécifique, richesses en genres, en familles, types morphologiques, diversité des habitats, degré de domestication) et les variables relatives aux connaissances et pratiques ethnobotaniques (nombre de maladies traitées, nombre de recettes ou d'usages rapportés, popularité des 
plantes, proportions d'utilisation des organes végétaux, des modes de préparation et des voies d'administration) en rapport avec le type de médecine (humaine ou vétérinaire), le test d'indépendance de Khi Deux de Pearson a été utilisé. Un modèle linéaire généralisé avec une analyse de covariance a permis de connaître globalement (les deux types de médecine) et spécifiquement (chaque médecine) les variables socioculturelles et démographiques qui déterminent la connaissance ethnobotanique (nombre de plantes mentionnées par individu par médecine) dans la zone d'étude. La variable « âge » représente la covariable et le

\section{RÉSULTATS}

Diversité des plantes utilisées dans les deux types de médecine : Au total, 90 espèces végétales à usages vétérinaires et 80 espèces utilisées en médecine humaine ont été recensées. La différence de connaissance relative à la richesse spécifique est très significative $(p=0,004)$. Cependant, il existe une très grande similarité $(86,69 \%)$ entre les deux types de médecine en termes d'espèces utilisées. La richesse en genres est plus élevée en médecine vétérinaire ( 81 genres) qu'en médecine humaine (75 genres) $(p=0,02)$. Néanmoins, $91 \%$ des genres recensés sont communs aux deux types de médecines. La richesse en familles est plus élevée en médecine vétérinaire (82 familles) qu'en médecine humaine (73 familles) $(p=0,001)$. Les familles les plus représentées en termes de nombre d'espèces sont les Leguminosae $(21,73 \%)$, Euphorbiaceae $(8,69 \%)$ et Combretaceae $(5,43 \%)$ pour la médecine vétérinaire et les Leguminosae $(14,13 \%)$, Euphorbiaceae $(8,69 \%)$ et Rubiaceae $(4,34 \%)$ pour la médecine humaine. Mais, la

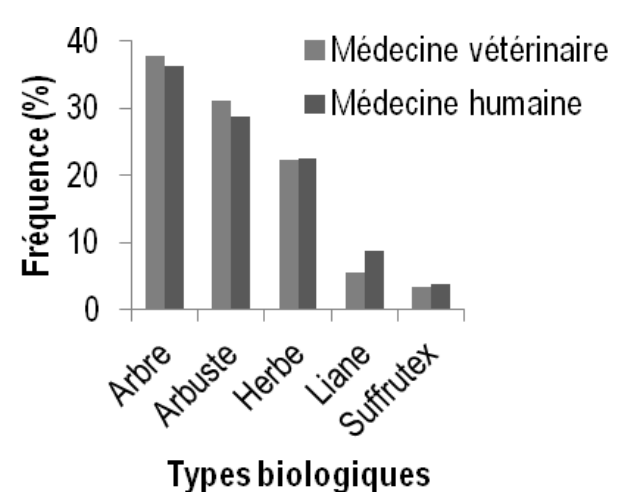

Figure 2 : Variation de la proportion d'utilisation des types morphologiques en fonction des types de médecine. test de Fisher a été utilisé pour déterminer la significativité de l'effet des variables. Afin de comparer les usages liés aux plantes impliquées dans les deux types de médecine, une fréquence a été calculée. II s'agit de la fréquence $(S)$ d'espèces ou d'usages communs aux deux médecines calculée suivant la formule : $S=\frac{\pi c}{\mathrm{~N}}$ où nc est le nombre d'espèces ou d'usages communs impliqués dans les deux médecines et $\mathrm{N}$ le nombre total d'espèces ou d'usages recensés. La similarité est : faible lorsque $S$ $<50 \%$; grande lorsque $50 \% \leq S<75 \%$; très grande lorsque $S \geq 75 \%$.

richesse en familles n'est pas significativement différente d'une médecine à l'autre (Chi2 $=4.0339, \mathrm{ddl}=45, \mathrm{p}=1$ ). Globalement, la majorité des espèces utilisées $(93,3 \%)$ poussent spontanément. En tenant compte des deux médecines, le constat est le même (92\% pour la médecine vétérinaire et $95 \%$ pour la médecine humaine). Malgré cela, la connaissance de l'état de domestication des plantes ne varie pas d'une médecine à l'autre (Chi2 = $0.0238, d d l=1, p=0.961$ ). Au total, 5 types morphologiques sont utilisés dans chaque type de médecine avec une prédominance des arbres $(37,8 \%$ en médecine vétérinaire et $36,25 \%$ en médecine humaine) (figure 2). Les moins utilisés sont les lianes et les suffrutex avec des taux respectifs de $8,75 \%$ et $3,75 \%$ en médecine humaine; et $5,6 \%$ et $3,3 \%$ en médecine vétérinaire. Mais, globalement, la connaissance relative au type morphologique important pour le soin est similaire entre les deux types de médecine (Chi2 $=0,88, d d l=4, p$ $=0,927$ ).

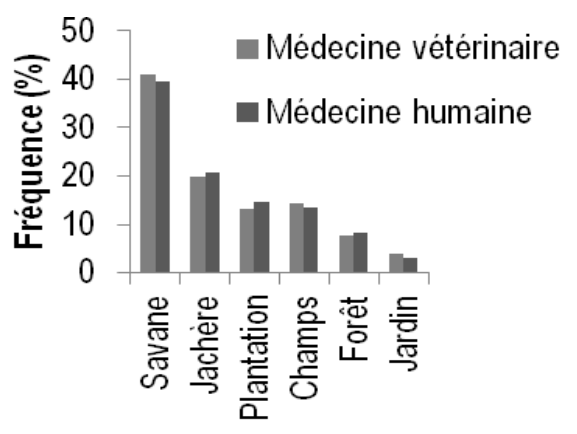

Habitats des espèces

Figure 3 : Variation de la fréquence des plantes récoltées par type d'habitat et par type de médecine. 
Les espèces recensées sont prélevées dans 6 habitats (figure 3). Les savanes hébergent la majorité des espèces, soit $41 \%$ du total pour la médecine vétérinaire et $40 \%$ pour la médecine humaine. Ensuite, viennent les jachères qui abritent respectivement $20 \%$ et $21 \%$ des espèces utilisées en médecines vétérinaire et humaine. Les champs abritent $14 \%$ des espèces pour les deux médecines et les plantations respectivement $13 \%$ et $15 \%$ des plantes à usages vétérinaires et humains. Néanmoins, il y a une similarité entre la proportion d'espèces dans chaque habitat entre les deux types de médecines (Chi2 $=0.215, \mathrm{ddl}=5, p=1$ ).

Pratiques traditionnelles dans les deux médecines : Au total, 13 maladies ont été recensées dont 10 sont communes aux deux types de médecine. Au regard de leur traitement, 110 recettes à usages vétérinaires ont été répertoriées et 86 à usages humains. La similarité entre les deux types de médecine est grande en termes de recettes indiquées (65\%). Le nombre de plantes entrant dans le traitement d'une maladie varie de 1 à 13 pour les deux types de médecine. Pourtant, le nombre de plantes impliquées dans le traitement des maladies ne dépend

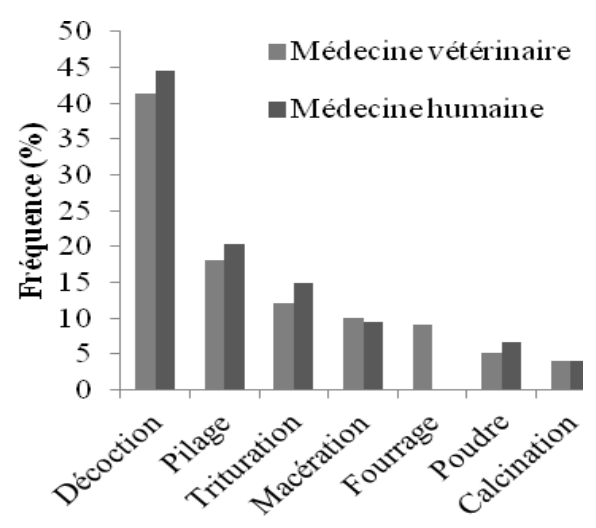

Modes de préparation

Figure 4 : Variation des modes de préparation des organes végétaux en fonction du type de médecine

En considérant la préparation des produits végétaux (figure 5), 7 modes pour la médecine vétérinaire et 6 pour la médecine humaine ont été recensés. La différence du nombre de mode de préparation entre les deux types de médecine n'est pas significative $(p=0,28)$. La fréquence de citation de ces modes varie de $4 \%$ pour le fourrage à pas du type de médecine au seuil de $5 \%$ (Chi2 $=0.7603$, $p=0.38)$. Les plantes les plus impliquées dans la composition des recettes en termes de popularité (nombre de citations) sont: Annona senegalensis, Bridelia ferruginea, Carica papaya, Securidaca longepedunculata, Xylopia aethiopica pour les deux types de médecine. Au nombre des espèces végétales traitant le plus grand nombre de maladies (au moins 5), on peut citer: Aframomum melegueta, Bridelia ferruginea et Momordica charantia pour les deux types de médecine. Toutefois, la connaissance relative à ces deux paramètres (popularité et nombre de maladies traitées) ne dépend pas du type de médecine au seuil de $5 \%$ (Chi2 $=6.6884 \mathrm{e}-31, \mathrm{ddl}=1, \mathrm{p}=1$ ).

Tous les organes végétaux sont sollicités dans les deux types de médecine. Les feuilles constituent l'organe le plus utilisé avec un taux de citation de $31,9 \%$ pour la médecine vétérinaire et $31,3 \%$ pour la médecine humaine (figure 4). Toutefois, la proportion d'utilisation des organes est similaire entre les deux types de médecines (Chi2 = 1,2207; ddl = 8; $p=0,996)$.

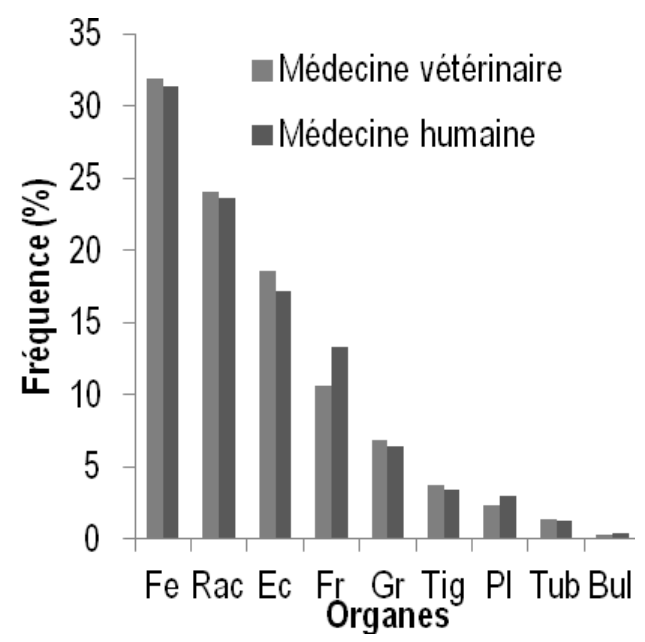

Légende : Fe : feuille ; Rac : racine ; Ec : écorce ; Fr : fruit ; $\mathrm{Gr}$ : grains; Tig : tige feuillée ; PI : plante entière ; Tub : tubercule; Bul : bulbe.

Figure 5 : Variation de la proportion des organes sollicités en fonction du type de médecine.

$41 \%$ pour la décoction dans le cas de la médecine vétérinaire et de $4 \%$ pour la calcination à $45 \%$ pour la décoction dans le cas de la médecine humaine. Mais cette variation n'est pas significative d'une médecine à l'autre (Chi2 = 7,318; $d d l=6 ; p=0,292$ ). En tenant compte de l'administration des produits, 2 voies ont été 
indiquées pour les deux types de médecine. Les applications internes (cumul des voies orale et anale) sont les prédominantes (figure 6) avec des taux de citation allant de $61 \%$ à $65 \%$ respectivement pour la médecine vétérinaire et la médecine humaine. Cette différence de proportion n'est pas significative $(p=0,74)$ et la connaissance relative au choix de la voie d'administration des médicaments traditionnels ne dépend pas du type de médecine (Chi2 $=2.5501 ; \mathrm{ddl}=2$; $p=0.2794$ ).

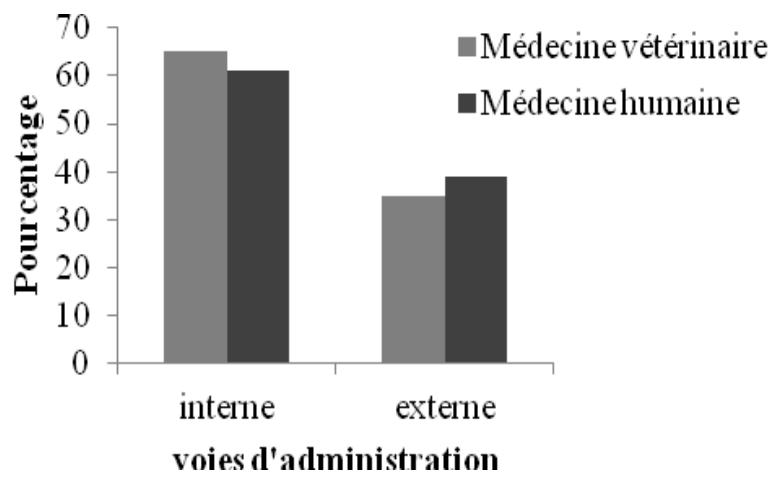

Figure 6 : Variation des voies d'administration des médicaments en fonction des médecines

En somme, la connaissance relative aux pratiques (maladies traitées, organes végétaux utilisés, modes de préparation et voies d'administrations des produits) n'est pas différente d'une médecine à l'autre.

Origine et déterminisme de la connaissance ethnobotanique dans les deux types de médecines : Pour $88 \%$ de réponses, les pratiques relatives à la médecine vétérinaire sont basées sur la connaissance en médecine humaine. Ceci stipule que la population enquêtée fait usage de sa connaissance dans le traitement des pathologies humaines pour la prise en charge des pathologies animales communes aux deux types de médecine. Toutefois, pour les $12 \%$ restants, c'est la médecine traditionnelle humaine qui a pris originellement source à partir du comportement thérapeutique naturel des animaux. La connaissance traditionnelle globale sur les plantes utilisées en ethnomédecine au Centre-Bénin ne varie pas significativement en fonction de l'âge, du sexe et de la profession $(p>0,05)$ (tableau 2). Toutefois, elle est influencée par l'appartenance sociolinguistique, le niveau d'instruction et l'origine de la connaissance dans la même aire géographique $(p<0,05)$. Le tableau 3 présente les déterminants de la connaissance ethnobotanique en fonction des médecines. II révèle qu'en médecine vétérinaire, l'appartenance sociolinguistique, l'âge, l'origine de la connaissance et le niveau d'instruction sont les variables qui influencent la connaissance liée à l'utilisation des plantes alors que l'appartenance sociolinguistique est le seul déterminant de la connaissance en médecine humaine au seuil de $5 \%$.

Tableau 2 : Résultats de l'analyse de covariance avec le modèle linéaire généralisé impliquant la connaissance globale en ethnomédecine

\begin{tabular}{l|c|c|c}
\hline Variables & ddl & F & P \\
\hline Ethnie & 3 & 3,38 & 0,02 \\
\hline Age & 1 & 0,48 & 0,49 \\
\hline Sexe & 1 & 0,72 & 0,39 \\
\hline Profession & 2 & 1,33 & 0,26 \\
\hline Niveau d'instruction & 3 & 2,54 & 0,05 \\
\hline Origine de la connaissance & 6 & 4,22 & 0,00 \\
\hline Erreur & 88 & - & - \\
\hline Total & 104 & - & - \\
\hline
\end{tabular}

ddl : degré de liberté ; F : valeur de Fischer ; $p$ : probabilité liée au test de Fischer 
Tableau 3 : Résultats de l'analyse de covariance avec le modèle linéaire généralisé impliquant la connaissance en fonction des deux médecines

\begin{tabular}{l|c|c|c|c|c}
\hline Variables & \multirow{2}{*}{ ddl } & \multicolumn{2}{|c|}{$\mathbf{F}$} & \multicolumn{2}{c}{$\mathbf{P}$} \\
\cline { 3 - 6 } & & MV & MH & MV & MH \\
\hline Ethnie & 3 & 3,86 & 6,85 & 0,015 & 0,005 \\
\hline Age & 1 & 14,08 & 2,10 & 0,0004 & 0,589 \\
\hline Sexe & 1 & 0,26 & 0,09 & 0,611 & 0,600 \\
\hline Profession & 2 & 1,98 & 3,05 & 0,149 & 0,075 \\
\hline Origine de la connaissance & 6 & 15,15 & 0,14 & $3,85 \mathrm{e}-8$ & 0,830 \\
\hline Niveau d'instruction & 3 & 6,80 & 0,67 & $6,84 \mathrm{e}-4$ & 0,643 \\
\hline
\end{tabular}

ddl : degré de liberté ; F : valeurs de Fisher ; P : Probabilité liée au test de Fisher. ; MV : Médecine Vétérinaire ; Médecine Humaine

Convergence de la connaissance ethnobotanique dans les deux médecines: Les richesses spécifiques les plus élevées (58 et 57 espèces) sont obtenues pour les Ifè respectivement pour les médecines vétérinaire et humaine (tableau 3). Cependant, la connaissance relative au nombre de plantes mentionnées par chaque ethnie était similaire entre les deux médecines (Chi2 $=3,849$; $d d l=3 ; p=0,278)$. Le nombre total d'usages rapportés est plus élevé chez les Ifè, Mahi et Peulh dans les deux médecines (tableau 3). Toutefois, la connaissance liée à ce paramètre n'est pas significativement différente d'une ethnie à l'autre dans les deux médecines (Chi2 $=4,286$; $\mathrm{ddl}=3 ; p=0,232$ ). Le tableau 3 indique aussi que le nombre de maladies traitées est plus élevé chez les Ifè, Mahi et Peulh dans les deux médecines. Pourtant, en considérant les deux médecines, la connaissance liée au nombre de maladies traitées ne dépend pas de l'ethnie (Chi2 = 0,503; ddl = 3; $p=0,918$ ). S'agissant du niveau d'instruction (tableau 4), les individus non scolarisés connaissent plus de plantes que ceux scolarisés en considérant chaque médecine. Néanmoins, ce niveau de connaissance est similaire entre les deux médecines (Chi2 $=0,592 ; d d l=1 ; p=0,442)$. Les plus forts taux $d u$ nombre d'usages rapportés (99 et 82 usages respectivement pour les médecines vétérinaire et humaine) ont été reportés pour les non instruits. Cependant, la connaissance liée aux usages rapportés n'est pas significativement différente dans les deux médecines (Chi2 $=1,878, d d l=1, p=0,171)$.

Tableau 4 : Étude comparative du niveau de connaissance intra et inter-ethnique entre médecines vétérinaire et humaine en fonction des ethnies.

\begin{tabular}{l|l|l|c|c|c|c}
\hline \multirow{2}{*}{ Ethnies } & \multicolumn{2}{|c|}{ Richesse spécifique } & \multicolumn{2}{c|}{ Nombre total d'usages } & \multicolumn{2}{c}{ Nombre de maladies traitées } \\
\cline { 2 - 7 } & $\mathrm{MV}$ & $\mathrm{MH}$ & $\mathrm{MV}$ & $\mathrm{MH}$ & $\mathrm{MV}$ & $\mathrm{MH}$ \\
\hline Idaatcha & $16^{\mathrm{c}}$ & $7^{\mathrm{f}}$ & $17^{\mathrm{h}}$ & $7^{\mathrm{k}}$ & $3^{\mathrm{m}}$ & $2^{\mathrm{o}}$ \\
Ifè & $58^{\mathrm{a}}$ & $57^{\mathrm{d}}$ & $48^{\mathrm{g}}$ & $47^{\mathrm{i}}$ & $10^{\mathrm{I}}$ & $10^{\mathrm{n}}$ \\
Mahi & $31^{\mathrm{b}}$ & $27^{\mathrm{e}}$ & $38^{\mathrm{g}}$ & $33^{\mathrm{j}}$ & $7^{1}$ & $9^{\mathrm{n}}$ \\
Peulh & $29^{\mathrm{b}}$ & $18^{\mathrm{e}}$ & $40^{\mathrm{g}}$ & $25^{\mathrm{j}}$ & $10^{\mathrm{l}}$ & $9^{\mathrm{n}}$ \\
\hline
\end{tabular}

MV : Médecine vétérinaire; $\mathrm{MH}$ : Médecine humaine ; Les valeurs portant les mêmes lettres en exposant dans une colonne ne diffèrent pas significativement au seuil de $5 \%$.

Le nombre de maladies traitées est similaire selon le niveau d'instruction (tableau 5). De plus, la connaissance liée à la prise en charge des maladies ne dépend pas $\mathrm{du}$ type de médecine $($ Chi2 $=0,020 ; \mathrm{ddl}=1 ; p=0,887)$.

Tableau 5 : Étude comparative du niveau de connaissance intra et inter-ethnique entre médecines vétérinaire et humaine en fonction du niveau d'instruction

\begin{tabular}{l|c|c|c|c|c|c}
\hline \multirow{2}{*}{ Instruction } & \multicolumn{2}{|c|}{ Richesse spécifique } & \multicolumn{2}{c|}{ Nombre total d'usages } & \multicolumn{2}{c}{ Nombre de maladies traitées } \\
\cline { 2 - 7 } & MV & MH & MV & MH & MV & MH \\
\hline non instruits & $72^{\mathrm{a}}$ & $64^{\mathrm{c}}$ & $99^{\mathrm{e}}$ & $82^{\mathrm{g}}$ & $12^{\mathrm{i}}$ & $11^{\mathrm{j}}$ \\
\hline instruits & $41^{\mathrm{b}}$ & $29^{\mathrm{d}}$ & $77^{\mathrm{f}}$ & $46^{\mathrm{h}}$ & $10^{\mathrm{i}}$ & $10^{\mathrm{j}}$ \\
\hline
\end{tabular}

MV : Médecine vétérinaire ; MH : Médecine humaine ; Les valeurs portant les mêmes lettres en exposant dans une colonne ne diffèrent pas significativement au seuil de $5 \%$. 


\section{DISCUSSION}

L'analyse des variables de la diversité floristique indique que la connaissance n'est pas plus diversifiée dans une médecine par rapport à l'autre. Ceci signifie qu'en tenant compte des paramètres floristiques, la connaissance ethnobotanique est globalement similaire entre les deux types de médecine. Ceci traduit une tendance vers le disponible thérapeutique à savoir que la plante utilisée dans le traitement d'une maladie humaine peut, dans une certaine mesure, traiter la même maladie chez les animaux et inversement. Des observations semblables ont été faites par Martínez et Luján (2011), dans leur étude sur la comparaison des deux types de médecine, bien que certaines espèces soient exclusivement utilisées dans la médecine humaine ou vétérinaire. De plus, les pratiques dans les deux types de médecine sont les mêmes au regard des organes végétaux utilisés, les modes de préparation adoptés et les voies d'administration privilégiées pour traiter une même maladie chez l'homme et chez l'animal. Ceci témoigne aussi d'une forte correspondance thérapeutique entre les deux types de médecine. Certains détails diffèrent cependant d'une médecine à l'autre. Quelques espèces ont été rapportées spécifiquement pour le traitement des maladies. C'est le cas par exemple de Cajanus cajan indiquée pour traiter la diarrhée uniquement chez les animaux et Cochlospermum planchoni, Bridelia ferruginea respectivement pour la diarrhée et les douleurs abdominales chez l'homme. L'appartenance sociolinguistique est le déterminant commun du niveau de connaissance dans les deux types de médecine. Ce constat est en rapport avec les travaux de Dassou et al. (2015) qui ont indiqué qu'au Bénin, l'appartenance d'un individu à un groupe sociolinguistique donné détermine son niveau de connaissance sur les ressources végétales à usages vétérinaires. L'appartenance sociolinguistique constitue donc le principal facteur car, selon Portères (1961), la naissance et le progrès des compétences

\section{CONCLUSION}

Au Centre-Bénin, les connaissances et pratiques ethnobotaniques sont identiques dans les médecines vétérinaire et humaine en termes de variables de diversité floristique et de celles relatives aux savoirs endogènes. Malgré la forte similarité entre les deux types de médecine, on ne pourrait affirmer que toutes les connaissances ethnobotaniques relatives à une médecine proviendraient de l'autre. L'hypothèse selon laquelle la culturelles sont basés sur les faits d'interrelations entre les ethnies et les plantes. Les autres facteurs (âge, niveau d'instruction, et origine de la connaissance) paraissent comme des déterminants secondaires du niveau de connaissance ethnovétérinaire. Ces constats montrent la singularité de la médecine vétérinaire par rapport à la médecine humaine. La connaissance de l'origine ou de l'histoire de la médecine vétérinaire traditionnelle africaine est un outil permettant de mettre en valeur la complémentarité qu'il existe entre les médecines traditionnelles humaine et vétérinaire. La présente étude a révélé les deux tendances c'est-à-dire que pour les uns, la médecine vétérinaire est née de la médecine humaine pendant que les autres enquêtés estiment que c'est la médecine humaine qui a pris source de la médecine vétérinaire. Toutefois, le premier courant est plus populaire et Lobry (2003) l'a confirmé dans son étude sur l'histoire des soins au bétail par les peulh, en indiquant qu'en Égypte, environ 5000 ans avant J.-C., les premiers médecins des hommes avaient été en même temps médecins des animaux. Néanmoins, la connaissance ethnovétérinaire peut constituer aujourd'hui un art contemporain acquis surtout par expérience ou test des plantes à usages humains sur les animaux. Ceci est d'une évidence particulièrement frappante au regard des considérations des guérisseurs enquêtés. La deuxième théorie qui n'a été abordée dans aucune étude scientifique mais est soutenue par beaucoup de témoignages, peut être aussi valable car encore de nos jours, certains herboristes essayent les remèdes sur des animaux domestiques, en particulier quand ils recherchent une toxicité. Ce travail n'est pas arrivé à confirmer l'une de ces idées mais considère que certaines connaissances ethnovétérinaires proviennent de la médecine humaine et que d'autres lui sont empruntées. D'où la grande similarité entre les deux types de médecine.

connaissance en ethnomédecine vétérinaire proviendrait de l'ethnomédecine humaine est donc rejetée. II existe simplement une complémentarité entre les deux types de médecine. En conséquence, les plantes efficaces utilisées en médecine vétérinaire pourraient être indiquées dans le traitement des mêmes maladies chez l'homme en cas d'une similarité d'agents pathogènes. 


\section{REMERCIEMENTS}

Les auteurs remercient l'Université d'Abomey-Calavi pour l'appui financier et témoignent leur gratitude à tous les éleveurs et guérisseurs qui ont participé aux enquêtes.

\section{RÉFÉRENCES BIBLIOGRAPHIQUES}

Akoègninou A., 2004. Recherches botaniques et écologiques sur les forêts actuelles du Bénin. Thèse d'État. Université de Cocody-Abidjan (Côte d'Ivoire), 326p.

Akoègninou A., van der Burg WJ., van der Maesen L.J.G, 2006. Flore Analytique du Bénin. Backhuys Publishers, Wageningen, Pays-Bas, 1034p.

Dassou GH, Ogni CA, Yédomonhan H, Adomou AC, Tossou M, Dougnon JT et Akoègninou A : 2014. Diversité, ethnobotanique et vulnérabilité des plantes à usages vétérinaires au Nord-Bénin. Int. J. Biol. Chem. Sci. 1(8) : 189-210.

Dassou GH, Yédomonhan H, Adomou AC, Ogni CA, Tossou GM et Akoègninou A, 2015. Facteurs socioculturels et environnementaux déterminant la connaissance ethnovétérinaire au Bénin. Afrique Science. 11 (5) : 335-360.

DE (Direction de l'Élevage), 2010. Annuaire statistique sur l'élevage. Direction de l'Élevage, Bénin, 0982.

Gnanglè PC, Égah J, Baco MN, Gbèmavo CDSJ, Kakaï RG et Sokpon N, 2012. Perceptions locales du changement climatique et mesures d'adaptation dans la gestion des parcs à karité au NordBénin. International Journal of Biological and Chemical Sciences 6(1): 136-149.

Guézodjè TGP, 2013. Étude ethnobotanique des plantes à usages vétérinaires au Bénin : cas du Plateau d'Abomey. Rapport de fin de formation pour l'obtention du diplôme de Licence Professionnelle. EPAC/UAC. 68 p.

INSAE (Institut National de la Statistique et de l'Analyse Économique), 2002. Troisième recensement général de la population et de l'habitat. Cotonou, Bénin. www.insae-bj.org

INSAE (Institut National de la Statistique et de l'Analyse Économique), 2013. Recensement Général de la Population et de l'Habitat (Résultats provisoires). Cotonou, Bénin.

Hounzangbé-Adoté SM, 2001. L'élevage face à la pharmacopée en médecine vétérinaire au sud du Bénin. Bulletin de la recherche agronomique $33: 1-9$.

Lans D, Harper T, Georges K, Bridgewater E, 2000. Medicinal plants used for dogs in Trinidad and Tobago. Prev. Vet. Med., 45: 201-220.
Lobry M, 2003. Les soins au bétail chez les peulhs : une tradition ancestrale. Bull. soc. Fr. Hist. méd. sci. vét. 2 (2).

Martínez JG, Luján MC, 2011. Medicinal plants used for traditional veterinary in the Sierras de Córdoba (Argentina): An ethnobotanical comparison with human medicinal uses. Journal of Ethnobiology and Ethnomedicine 7:23.

McCorkle C, Martin M, 1998. Parallels and potentials in animal and human ethnomedical technique. Agric. Human Values, 15: 139-144.

Portères R, 1961. L'ethnobotanique: Place-ObjetMéthode-Philosophie. Journal d'agriculture tropicale et de botanique appliquée. 8, 102-109.

Scarpa GF, 2000. Plants employed in traditional veterinary medicine by the criollos of the Northwestern Argentinean Chaco. Darwiniana, 38, 253-265.

Schillhorn van Veen TW, 1998. One medicine: The dynamic relationship between animal and human ethnomedical technique. Agric. Human Values, 15, 115-120.

Tamboura H, Kaboré H, Yaméogo SM, 1998. Ethnomédecine vétérinaire et pharmacopée traditionnelle dans le plateau central du Burkina Faso: cas de la province du Passoré. Biotechnologie, Agronomie, Société et Environnement 2 (3), 181-191.

van den Veerle E, van Damme EP et De Wolf J, 1993. Inventaire et modelage de la gestion du couvert pérenne dans une forestière du sud du Sénégal. Rapport final, Partie C : Étude ethnobotanique. Université de Gent. Belgique, 102 p. 